\title{
Size Dependent Properties of Hollow Gold Nanoparticles: A Theoretical Investigation
}

\author{
S. Senturk Dalgic* \\ Trakya University, Faculty of Science, Department of Physics, Balkan Campus 22030 Edirne, Turkey
}

\begin{abstract}
A new kind of nanostructures with the negative curvature defined as the hollow ones have recently used in biomedical applications. In this work, an analytic model was developed to compute the size-dependent properties of spherical hollow gold nanoparticles in shell-core-shell configuration. This model has established to calculate the cohesive energies based on the surface energy consideration depending on sizes of inner and outer surfaces of hollow nanoparticles. The size and geometry of the model particles have been obtained by using the stability diagram and the collapsing mechanism was studied by molecular dynamics simulations. The model has been also applied to the hollow particles within unstable and half stable geometry. The predicted results have been compared with each other and those obtained by solid ones. The theoretically predicted size dependent properties are consistent with experimental observations and the hollow quantum dot calculations. Thus, an atomistic insight into the size effect on the cohesive energies of hollow nanoparticles has been presented.
\end{abstract}

DOI: 10.12693/APhysPolA.129.531

PACS/topics: 81.07.Bc, 82.60.Qr, 82.20.Wt

\section{Introduction}

Hollow nanostructures have attracted considerable attention in many research areas, including chemical or biological sensors, catalysis, drug delivery and optoelectronic applications [1]. They show unusual properties by comparing with the nanostructures of positive curvature such as nanoparticles and quantum dots. Recent applications of nanomaterials have focused on gold nanostructures. Among them, gold hollow nanoparticles have fascinating properties because of their attractive surface plasmon resonance (SPR) feature which makes them optically active nanostructures of considerable interest in both fundamental research and practical applications [24]. Researchers have noted that gold nanoparticles can be designed to absorbed light near-infrared range (NIR) which is innocuous to the human tissue [2]. Subsequently, hollow gold nanospheres (HGNs) have emerged as an excellent candidate for cancer imaging and therapy. It has observed that the characteristic SPR peaks of HGNs show an evident blue shift from one HGN to another one due to the increase of shell thickness [3]. Schwartzberg and co-workers have also reported the varying of optical properties of HGNs in sizes between 20 and $70 \mathrm{~nm}$ by geometric parameters of shell thickness and cavity size related with their thermal and mechanical stability [4]. It may be noted that modulating and controlling of these parameters are also dependent on the method used for the synthesis of the nanoparticles. However, another significant phenomenon is possible to observe that the hollow nanostructures similar to nanovoids and nanocavities may become shrinkage or collapse at elevated temperatures which strongly affect their physical and chemical properties [5-7].

*e-mail: serapd@trakya.edu.tr; dserap@yahoo.com
The hollow nanospheres are complex structures apparently obtained in experiments. Therefore theoretical studies could help to understand the thermal stability behaviors of hollow nanoparticles (HNPs). Molecular dynamics (MD) simulations are also a powerful tool to correlate the geometric parameters with the material stability instead of the direct material synthesis [8-11]. Schwarz and Safran reported first the size dependent material properties of multiwall HNPs [12]. Jiang and coworkers have investigated the thermal stability of hollow gold nanoparticles (HGNPs) with core-shell structured using MD simulations to obtain the stability diagram based on the aspect ratio (the ratio of outer radius over shell thickness) and collapsing mechanism [6, 7].

Despite the theoretically predicted thermal stability diagram of HGNPs, a detailed knowledge of the thermodynamic behaviors of hollow particles is still lacking. According to our knowledge, there is not any theoretical investigation based on an atomic insight into the underlying mechanism to explain the thermal behaviors of HGNPs in geometrically shell-core-shell structure with a surface shell of an atomic diameter. The primary aim of this work is to present an analytic model for the sizedependent properties of hollow gold metallic nanoparticles within diameters of 3-15 $\mathrm{nm}$ which can be easily implemented in MD simulations.

In this work, an analytic model was established for understanding the size effect on the cohesive energy of HGNPs regarding average coordination numbers based on the surface energies in conjunction with the bond-order-length-strength (BOLS) correlation mechanism [13] and the structure model proposed for hollow quantum dots (HQDs). However, in this contribution, unlike these two models, (i) the bond contraction $c_{i}$ has been considered only for the first atomic layers of inner and outer shells, (ii) the elastic energy contribution to the surface energy is ignored in our calculations. 
The calculated cohesive energies modeled for HGNPs have been compared with those obtained by MD calculations.

Here, we show with this model that first atomic layer of both surfaces in hollow nanoparticles is sufficiently enough to describe the size effect on the bond length contraction, surface energy and cohesive energy of particles which are of diameters $>3 \mathrm{~nm}$.

\section{Materials and method}

The hollow structure has been adopted as a core-shell type with the cavity surrounded by the core. In the present case, the shell-core-shell configuration of an HGNP with $N$ atoms located on inner and outer surfaces was considered. The schematic illustration of the shell-core-shell structural model of the hollow system is shown in Fig. 1. The size-dependent properties of hollow nanoparticles at a given temperature have been made to explain with an analytical model which can apply to the MD simulations.

The derivation of the model is as follows: Starting with the surface to volume ratio of an $i$-th atomic layer, $\xi_{i}$ is given by $\xi_{i}=\frac{N_{i}}{N}$. The subscript $i$ denotes the atom in the $i$-th atomic layer. Therefore, considering the number of atoms in inner and outer surfaces with $N_{\text {in }}$ and $N_{\text {out }}$, the ratios for inner and outer skins are $\xi_{\text {in }}$ and $\xi_{\text {out }}$, respectively, as follows:

$$
\xi_{\text {in }}=\frac{3 h}{R} \frac{\left(\frac{r_{0}}{R}\right)^{2} c_{\text {in }}}{1-\left(\frac{r_{0}}{R}\right)^{3}}, \quad \xi_{\text {out }}=\frac{3 h}{R} \frac{c_{\text {out }}}{1-\left(\frac{r_{0}}{R}\right)^{3}},
$$

where $h$ is the diameter of an atom, $c_{\text {in }}$ and $c_{\text {out }}$ represent the bond contraction coefficients for negatively and positively curved surfaces, respectively. In the BOLS correlation, $c_{i}$ describes the changes in the bond length of an atom by including the coordination number $(\mathrm{CN}) z_{i}$ in the $i$-th atomic layer counted from the surface. It has been proposed that the $\mathrm{CN}$ imperfection of an atom on a surface causes the remaining bonds of the lowercoordinated surface atom to relax spontaneously [13-15]. The bond contraction coefficient $c_{i}$ is represented regarding bond length and bond strength correlation as

$$
c_{i}\left(z_{i}\right)=2 /\left[1+\exp \left(\left(12-z_{i}\right) / 8 z_{i}\right)\right] \text {. }
$$

Recent studies show that the first or two, atomic layers normal to the metallic surface is sufficient for describing the surface contraction [15]. Recently, Huang and co-workers have studied the surface structures of $\mathrm{Au}$ nanocrystals of 3 to $5 \mathrm{~nm}$ in diameters by coherent diffraction experiments and observed that the bond contraction was around $0.05 \AA$ for atoms in the middle of the $\{111\}$ facets. On this line, the one single atomic layer of surface shell of two sides of HGNP is taken into account. Thus, the CNs of an atom in negatively and positively curved surfaces of hollow nanoparticles can be expressed in the light of the BOLS mechanism as the following form, respectively.

$$
z_{\text {in }}=4\left(1+1.5 h /\left(2 r_{0}\right)\right), \quad z_{\text {out }}=4[1-1.5 h /(2 R)] .(3)
$$

On the other hand, an atom placed in the core region is considered as the bulk atom (or interior atom). The CN of atoms in the lattice for the bulk atom is equal to 12 for the face-centered-cubic (fcc) lattice. From the fundamental point of view, the atomic cohesive energy of an atom $i,\left(E_{i}\right)$, can be formulated as $E_{i}=E_{\mathrm{COH}}^{\mathrm{B}} / z_{i}$, where $E_{\mathrm{COH}}^{\mathrm{B}}$ is the bulk cohesive energy. It has been reported the cohesive energy of an atom $E_{i}$ in small clusters; $E_{i}=E_{\mathrm{COH}}^{\mathrm{B}}\left(z_{i} / z_{\mathrm{b}}\right)^{1 / 2}$ where $z_{\mathrm{b}}$ is the $\mathrm{CN}$ of an atom in bulk. Furthermore, it has been noted that the cohesive energy of surface atom $E_{\mathrm{COH}}^{\mathrm{S}}$ related to the cohesive energy of interior atom as $E_{\mathrm{COH}}^{\mathrm{S}}=z_{\mathrm{e}, i} / z_{\mathrm{b}} E_{\mathrm{COH}}^{\mathrm{B}}$, where $z_{i \text { e }}$ an effective $\mathrm{CN}$ (ECN) of the atom $i$. In their relation surface and bond contraction such as bond order, bond length, and surface relaxation have been also considered. Hence, the average ECN $\left(\bar{Z}_{\mathrm{e}, N}\right)$ of hollow nanoparticle containing $N$ atoms with shell-core-shell structure is considered as

$$
\bar{Z}_{\mathrm{e}, N}=z_{\mathrm{b}}\left(\xi_{\text {in }} z_{\text {in }, \mathrm{b}}+\xi_{\text {out }} z_{\text {out }, \mathrm{b}}+\xi_{\text {core }}\right) .
$$

According to the shell-core-shell structure of hollow nanoparticles, the average cohesive energy can be given by

$$
\begin{aligned}
& \left\langle E_{\mathrm{COH}}\left(R, r_{0}\right)\right\rangle=N_{\text {in }} E_{\mathrm{COH}}^{\text {in }}+N_{\text {out }} E_{\mathrm{COH}}^{\text {out }} \\
& \quad+\left(N-N_{\text {in }}-N_{\text {out }}\right) E_{\mathrm{COH}}^{\mathrm{B}} .
\end{aligned}
$$

On the other hand, the surface free energy of the negatively and positively curved surfaces of the hollow shellcore-shell structure was given by concerning surface $z_{\mathrm{s}}$ and bulk $z_{\mathrm{B}}$ coordination number as $\gamma=\gamma_{\text {in }}+\gamma_{\text {out }}$. The chemical part of the surface free energy is $\gamma=$ $\gamma_{0}(1 \pm 1.45 h / D)$, where $\gamma_{0}=\left(1-\sqrt{z_{\mathrm{s}} / z_{\mathrm{B}}}\right) E_{\mathrm{COH}}^{\mathrm{B}}$. It has been noted that the strained surface free energy is small compared to the chemical part that can be ignored in our calculations [16].

\section{Results and discussion}

Using established model, the size-dependent cohesive energies, bond contraction coefficient in inner and outer surfaces have been calculated. The capability of the proposed model has been tested by computing the sizedependent cohesive energies, diffusion coefficients of different hollow structures by MD simulations. The hollow structure has been adopted as core-shell type nanoparticles with the cavity surrounded by the core. In the present case, we consider the shell-core-shell configuration of a hollow gold nanosphere in our calculations as shown in Fig. 1a and the corresponding one-half of the MD sample is illustrated in Fig. 1b. In this work, the $3 \mathrm{D}$ atomic models of hollow nanoparticles are created through a top-down method in MD calculations. First, a solid sphere is extracted from a bulk fcc Au crystal with the [100], [010] and [001] crystallographic directions using a series of spherical cutoff centered at a core of cubes. Then, removing atoms from the center of the nanoparticle leaves a cavity space inside and thus forms a hollow nanoparticle, as indicated in Fig. 1b. The hollow system has been attributed to as inner-outer radius using the gold crystal lattice parameter, $0.4065 \mathrm{~nm}$. The outer and inner diameter, $K$ and $L$ are the dimensionless forms of size representing the number of crystal cells lined along 
the radius of the sphere and the hollow void, respectively. The only one single atomic layer with the surface of two sides of HGNs is taken into account.

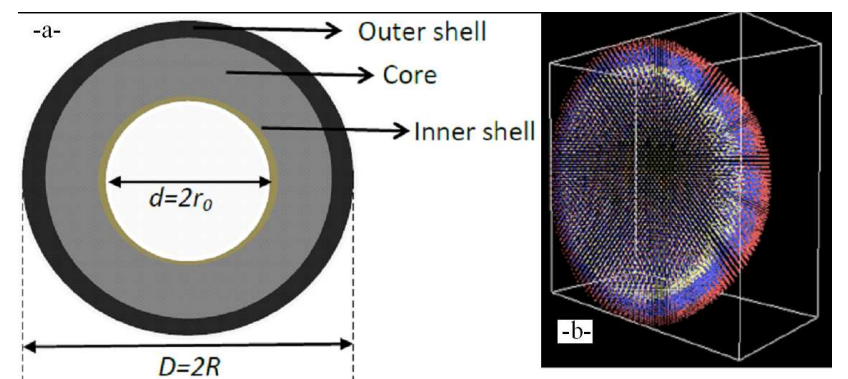

Fig. 1. (a) A schematic illustration of the shell-coreshell structure model and (b) HGNP used in MD simulations with one-half cut from the sample.

In our MD calculations, the quantum corrected Sutton-Chen (Q-SC) type potentials [11] were adopted to describe the interatomic interactions in HGNPs for different sizes. These potentials represent many-body interactions, and their parameters have been optimized according to the lattice parameter, cohesive energy and bulk modulus of gold. Possible parameters used in atomistic simulations can be found elsewhere [11]. DLPOLY simulation code establishes MD simulations [17]. The bulk system is performed with molecular dynamics simulations under constant temperature and constant pressure condition (NPT). The nanoparticles are simulated with constant temperature and constant volume (NVT) molecular dynamics without the periodic boundary conditions.

All the nanoparticles became the stable state after the enough steps to reach the potential plateau. Brendsden thermostat was applied in NVT-MD simulations with 0.5 values. The leapfrog Verlet velocity algorithm was integrated with the equations of atomic motions. The time step of simulations is $0.025 \mathrm{ps}$. The MD simulations have been carried out in 300000 steps and during atomic coordinates, velocities and energies were extracted for the statistical quantities in the last 200000 steps.

The calculated size-dependent cohesive energies are shown in Fig. 2a along with outer diameter for different hollow thicknesses. Figure $2 \mathrm{~b}$ also shows the sizedependent cohesive energies at a constant outer radius.

The values at $r_{0}=0$ in Fig. $2 \mathrm{~b}$ are corresponding to the value of solid nanoparticles. Notably, as seen in Fig. 2b, $E_{\mathrm{COH}}$ decreases when the size is reduced. This trend is observed in MD simulations as shown in Fig. 3 and in agreement with the other theoretical calculations obtained by Jiang et al. [7]. Figure 3a shows the predicted results due to different aspect ratios up to 5 .

Jiang et al. [7] have created the diagram of the twodimensional phase space of the thermal stability using MD simulations at a temperature of $300 \mathrm{~K}$. During the relaxation process, the HGNPs are classified into three categories, i.e., unstable, half-stable, and stable systems.

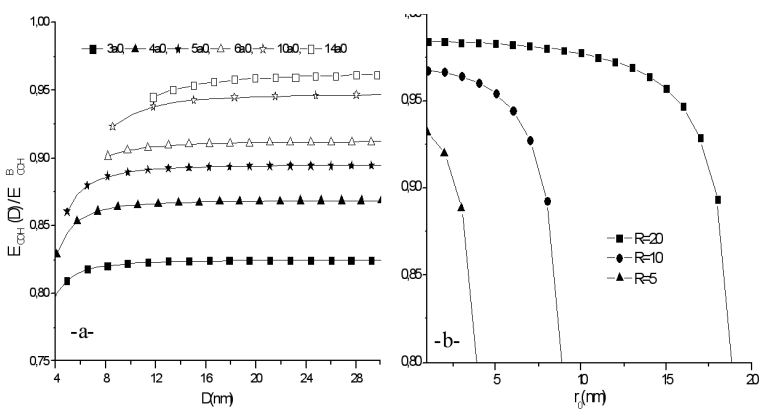

Fig. 2. Size-dependent cohesive energies of HGNPs versus (a) outer diameter for different hollow thicknesses regarding $a_{0}$ parameter and (b) inner radius at three various sizes of HGNPs.

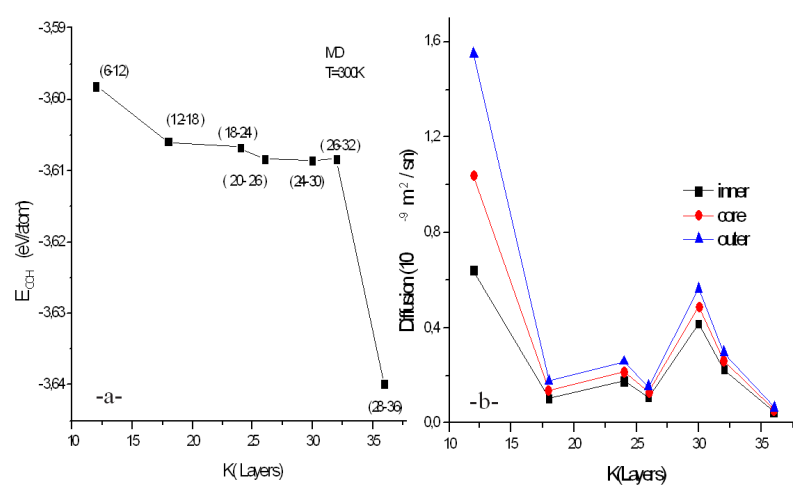

Fig. 3. (a) Size-dependent cohesive energies and (b) self-diffusion coefficients obtained by MD simulations for HGNPs versus $K$ layers corresponding to the outer radius.

There is no unstable system when the wall thickness is larger than $2.0 \mathrm{~nm}$. According to our model structure, there are no unstable and semistable hollow particles. Our results seen in Fig. 3a, agree with Jiang et al. that $E_{\mathrm{COH}}$ decreases when the aspect ratio increases. These are due to the more stable particles than others. When the wall thickness stays constant, HGNP is not half stable in this model structure.

However when the aspect ratio is around 6, some small vacancies are observed in MD simulations at $300 \mathrm{~K}$. When aspect ratio is around 2, octahedral shapes of hollow nanoparticles have been obtained, because the atoms have been coordinated like as atomic clusters at that size. Jiang et al. [7] noted that when the wall is thicker than 2.5 , the system moves from the stable region to the half stationary part in the two-dimensional phase space of the thermal stability. They have predicted, when the wall thickness is constant, that the larger hollow nanoparticle becomes more unstable structure. If we create the hollow particles as shell-core-shell structure, the particles will become more stable when the wall thickness is greater. That is related with the surface melting of HGNPs. Figure $3 \mathrm{~b}$ shows the corresponding self-diffusion coefficients of the MD samples placed in Fig. 3a. It is clear that the surface melting begins from the outer surface at all HGNPs. However, the outer surface of HGNPs with a 
diameter smaller than $10 \AA$ has just melted at $300 \mathrm{~K}$. It has found that the calculated cohesive energies for solid nanoparticles have been more stable than hollow ones, because HGNPs have greater energies than solid spherical nanoparticles at the same sizes. These results agree with other theoretical calculations and MD simulations.

\section{Conclusion}

In this work, an analytical model is presented to determine the size-dependent cohesive energies of HGNPs. The predicted results also agree with HQD calculations and provide a fundamental perspective for HGNPs with a diameter of 3-15 nm. The shell-core-shell structure for hollow nanoparticles gives a better description of thermal and mechanical stability than core-shell structured models. This model was applied to MD simulations to obtain the thermodynamics and physical properties of free-standing hollow nanoparticles. The thermal stability diagram using the proposed model for HGNPs is also established which could be useful to experimental synthesis. This model will extend to the hollow nanoparticles embedded in a matrix which is important in biomaterial engineering researches.

\section{References}

[1] E. Lim, T. Kim, S. Paik, S. Haam, Y. Huh, K. Lee, Chem. Rev. 115, 327 (2015).

[2] S. Adams, D. Thai, X. Mascona, A.M. Schwartzberg, J.Z. Zhang, Chem. Mater. 26, 6805 (2014).

[3] H. Liang, L. Wan, C. Bai, L. Jiang, J. Phys. Chem. B 109, 7795 (2005).
[4] A.M. Schwartzberg, T.Y. Olson, C.E. Talley, J.Z. Zhang, J. Phys. Chem. B 110, 19935 (2006).

[5] X. Bai, M. Li, Nano Lett. 6, 2284 (2006).

[6] L. Jiang, X. Yin, J. Zhao, Chin. J. Inorg. Chem. 25, 176 (2009).

[7] L. Jiang, X. Yin, J. Zhao, H. Liu, Y. Liu, F. Wang, J. Zhu, F. Boey, H. Zhang, J. Phys. Chem. C $\mathbf{1 1 3}$, 20193 (2009).

[8] F. Delogu, J. Phys. Chem. A 112, 2863 (2008).

[9] S. Senturk Dalgic, U. Domekeli, J. Optoelectron. Adv. Mater. 11, 2126 (2009).

[10] S. Senturk Dalgic, J. Optoelectron. Adv. Mater. 11 , 2133 (2009)

[11] R. Huang, G. Shao, X. Zeng, Y. Wen, Sci. Rep. 4, 7051 (2014)

[12] U.S. Schwarz, S.A. Safran, Phys. Rev. E 62, 6957 (2000).

[13] C.Q. Sun, Progr. Solid State Chem. 35, 1 (2007).

[14] G. Ouyang, G.W. Yang, Appl. Mater. Interf. 4, 210 (2012).

[15] W.J. Huang, R. Sun, J. Tao, L.D. Menard, R.G. Nuzzo, J.M. Zuo, Nature Mater. 7, 308 (2008).

[16] S. Xiong, W. Qi, Y. Cheng, B. Huang, M. Wang, Y. Li, Phys. Chem. Chem. Phys. 13, 10648 (2011).

[17] DL POLY: a molecular dynamics simulation package was written by W. Smith, T.R. Forester, I.T. Todorov obtained from the website www.ccp5.ac.uk/DL_POLY. 